\title{
CALIDAD DE VIDA, ESTADO EMOCIONAL, ANEMIA Y ASTENIA EN PACIENTES DE CÁNCER DE COLON TRATADOS CON CIRUGÍA
}

\author{
QUALITY OF LIFE, EMOTIONAL STATE, ANEMIA, AND FATIGUE IN COLON CANCER \\ PATIENTS AFTER SURGERY
}

\author{
Sonia López-Santiago', Juan A. Cruzado', Virginia Martínez², Jaime Feliv² \\ Facultad de Psicología. Universidad Complutense de Madrid \\ 2 Servicio de Oncología Médica. Hospital Universitario la Paz de Madrid
}

Resumen

Objetivo. El cáncer y sus tratamientos pueden alterar la adaptación del paciente a su vida cotidiana. Este trabajo pretende indagar el impacto del cáncer de colon y su tratamiento con cirugía en la calidad de vida, el estado de ánimo, astenia y anemia en una muestra de 77 participantes.

Método: estudio descriptivo transversal con evaluación de calidad de vida (QLQ-C30), ansiedad y depresión (Escala de Ansiedad y Depresión Hospitalaria), astenia (Breve Inventario de Fatiga), anemia (hemoglobina $<12$ g/ dl. en mujeres y $<13$ g/dl. en hombres).

Resultados: el $6,5 \%$ obtiene una puntuación de ansiedad dentro del rango clínico, el $7,8 \%$ una puntuación en depresión dentro del rango clínico. Como promedio, el grupo de mujeres tiene $11,9 \mathrm{~g} / \mathrm{dl}$. de hemoglobina en sangre, los hombres tienen $12,65 \mathrm{~g} / \mathrm{dl}$. La puntuación en astenia media es de 1,35 sobre 10 puntos y en interferencia de la astenia con la vida diaria de 0,85 sobre 10 . Respecto a los datos europeos de pacientes de cáncer de colon local, la calidad de vida global de los evaluados es buena $(69,26$; Dt 24,45), las áreas funcionales satisfactorias, tres de ellas superiores al valor de referencia (física, rol y social), y los síntomas disnea y dolor son inferiores a dichos valores.

Conclusiones: la alteración de aspectos bio-psico-sociales en pacientes de cáncer de colon tratados con cirugía se limita a un por-
Abstract

Objective: Cancer and its treatments can alter the patient's adaptation to their daily lives. This work aims to investigate the impact of colon cancer and surgery treatment in quality of life, emotional state, fatigue and anemia in a sample of 77 patients.

Methods: Cross-sectional descriptive study assessing quality of live (QLQ-C30), anxiety and depression (Hospital Anxiety and Depression Scale), fatigue (Brief Fatigue Inventory), anemia (hemoglobin $<12 \mathrm{~g} / \mathrm{dl}$. for women and $<13 \mathrm{~g}$ / dl. for men).

Results: Mean scores for anxiety and depression were within the normal range, $6.5 \%$ obtained a score of anxiety within the clinical range, and $7.8 \%$ a depression score within the clinical range. On average, the group of women is $11.9 \mathrm{~g} / \mathrm{dl}$. blood hemoglobin, men are $12.65 \mathrm{~g} / \mathrm{dl}$. The average fatigue score of 1.35 out of 10 points and fatigue interference with daily life of 0.85 out of 10. Regarding the European data of patients with local colorectal cancer, the overall quality of life of the individuals was high (69.26, Dt 24.45), satisfactory functional areas, three above the reference value (physical, social and role), and dyspnea and pain symptoms are lower than these levels.

Conclusions: Only a small percent of colon cancer patients have some impairment in biological, psychological and social areas explored. The scores in quality of life of cancer

\section{Correspondencia:}


centaje reducido. La calidad de vida del paciente oncológico español es muy similar al baremo europeo propuesto e incluso mejor en algunas áreas funcionales y síntomas.

Palabras clave: Calidad de vida, ansiedad, depresión, astenia, anemia, cáncer de colon.

\section{INTRODUCCIÓN}

El cáncer representa la segunda causa de muerte tanto en la población general española como en el subconjunto de mujeres españolas, siendo las enfermedades cardiovasculares la primera causa de mortalidad. En el subconjunto de hombres constituye la primera causa de muerte, la segunda son las enfermedades del corazón.

El cáncer de colon es una proliferación celular anómala localizada en cualquier tramo del colon, debida a mutaciones genéticas que favorecen la evitación de la apoptosis y potencian la capacidad de metastatizar $^{(1)}$. Este tipo de cáncer se ubica en el segundo lugar en mortalidad, tanto en hombres como en mujeres, tras el cáncer de pulmón y el de mama respectivamente. El número de defunciones en España en el año 2008 causadas por esta neoplasia fue de 10.583, a una edad media de 72 años, teniendo lugar el $56 \%$ de ellas en hombres $^{(2)}$. Su aparición es más probable en varones mayores de 50 años.

Vista la elevada mortalidad asociada al cáncer en general y al cáncer de colon en particular, no es de extrañar que su diagnóstico suponga un evento vital estresante, pese a la mejora en los tratamientos y la prolongación de la supervivencia, el miedo a la muerte, el shock emocional inicial, pueden poner en peligro el equilibrio mantenido entre las esferas bio-psicosociales de la vida del paciente y de las personas de su entorno cercano.

Tanto la enfermedad, como el diagnóstico, así como los tratamientos empleados para prolongar la supervivencia de patients In Spain is very similar to European reference values and even better in some functional areas and symptoms.

Key words: Quality of life, anxiety, depression, fatigue, anemia, colon cancer.

los pacientes pueden tener consecuencias negativas para la adaptación a la vida cotidiana. La vida del paciente puede verse alterada por el deterioro físico, emocional y social, debido a diferentes factores como pueden ser: un incremento de la fatiga, el desarrollo de anemia severa, ansiedad, depresión y alteraciones en la calidad de vida. Además, dado el envejecimiento de la población española y la alta incidencia y prevalencia del cáncer de colon en personas mayores, más vulnerables, se acrecienta el interés por su estudio ${ }^{(3)}$. Por todo ello, este trabajo explora el impacto en dichas variables, en pacientes que han sido diagnosticados recientemente de cáncer de colon que ha sido extirpado mediante tratamiento quirúrgico.

\section{MÉTODO}

\section{Muestra}

Los criterios de inclusión que debían cumplir los pacientes seleccionados para el presente estudio fueron: ser mayor de 18 años; estar diagnosticado de cáncer de colon (estadio II o III), con tratamiento quirúrgico, prestar libre y voluntariamente su consentimiento informado para ser partícipe de la investigación, tener comprensión y expresión adecuada del castellano.

Los participantes no debían cumplir ninguno de los criterios de exclusión: ser mayor de 85 años; estar diagnosticado de un estadio I (tumor confinado a la mucosa y submucosa) o de un estadio IV (presencia de metástasis a distancia), enfermedad neurológica, trastorno psiquiátrico grave, adicción a drogas o medicación psicoacti- 
va, tratamiento local o sistémico concomitante (radioterapia, hormonoterapia).

Se solicitó su contribución a un total de 83 pacientes que cumplían todos los criterios de inclusión y ninguno de exclusión, que en un primer momento accedieron a colaborar. De los participantes que aceptaron, cuatro se ausentaron por problemas de salud y otros dos retractaron el consentimiento informado por baja motivación, estos seis pacientes constituyen un $7,3 \%$ de pérdida de participantes en la investigación. La muestra definitiva se compone de 77 participantes, cuyas características se muestran en la tabla 1.

\section{Procedimiento}

El equipo de oncología médica del Hospital Universitario La Paz de Madrid reclutó a una serie de pacientes de cáncer de colon de reciente diagnóstico tratados con cirugía (principalmente sigmoidectomía y hemicolectomía).

Los profesionales del equipo informaban a los pacientes que cumplían los requisitos sobre la investigación, invitándolos a participar y solicitando un consentimiento informado escrito emitido libre y voluntariamente. Los 77 pacientes que accedieron fueron evaluados una media de mes y medio tras el tratamiento quirúrgico. En la cita convenida se extraía una muestra de sangre, se daba información médica pertinente y posteriormente se aplicaban las pruebas de autoinforme seleccionadas para la medición de las variables de interés.

\section{Variables e Instrumentos de medida}

Calidad de vida: se evaluó la calidad de vida global, cinco áreas funcionales (física, rol, cognitiva, social y emocional) y los síntomas que puede alterarla ${ }^{(4-9)}$.

Estado de ánimo: se evaluó el grado de ansiedad y depresión de los pacientes ${ }^{(10-12)}$.
Astenia: se examinó la presencia o ausencia de astenia general en el paciente así como la interferencia que ésta puede causar en diferentes aspectos de la vida diaria(13-15). $^{(12}$.

Anemia: se midió mediante la cantidad de hemoglobina en sangre empleando para ello un hemograma.

Los instrumentos empleados para el estudio de las variables de interés se pueden observar en la tabla 2 .

\section{Análisis estadístico}

Para llevar a cabo el análisis estadístico se empleó el programa SPSS (versión 17.0).

Se obtuvieron datos descriptivos tales como media, mediana, moda, para variables cuantitativas y frecuencias absolutas, porcentajes para variables cualitativas.

Además, la puntuación promedio en calidad de vida global, áreas funcionales, escalas sintomáticas y síntomas que pueden alterar la calidad de vida se compararon con los datos normativos de la población europea de referencia con cáncer de colon local, empleando para ello la prueba $t$ de Student.

El intervalo de confianza para aceptar la hipótesis nula fue del 95\%.

En el caso de los aspectos emocionales (ansiedad y depresión), astenia, y anemia, se obtuvieron las puntuaciones promedio de la muestra, valorando su pertenencia a rangos considerados normales o clínicos.

Para la ansiedad y la depresión se realizó un análisis de la puntuación promedio y del porcentaje de casos no clínicos, borderline y clínicos tras la cirugía.

\section{RESULTADOS}

Las características principales de la muestra son:

- Datos sociodemográficos: varón $(63,6 \%)$ de 67 años (media $=67,6$; 


\section{Tabla 1. Características de la muestra examinada}

$\mathrm{N}=77 \quad$ Media Mediana Dt Mín-máx. Frecuencia Porcentaje

$\begin{array}{lllll}\text { Edad } & \mathbf{6 7}, \mathbf{6 0} & 67,00 & 9,19 & 38-85\end{array}$

Genero:

Hombres

Mujeres

28

36,4

Estado civil:

Soltería

Divorcio

Matrimonio

61

Viudedad

Pareja de hecho

Convive en:

Soledad

$$
6
$$

Pareja

Hijos

Otros

Profesión:

Tareas del hogar

Trabajo por cuenta propia

Funcionariado

Trabajo por cuenta ajena

Otros

Situación laboral actual:

En activo

Baja laboral transitoria o permanente

Jubilación

Otros

Años de escolaridad:

Menos de 5 años

Entre 5 y 12 años

Más de 12 años

Dominancia manual:

Mano derecha preferente

Mano indiferente

Antecedentes neuropsicológicos familiares:

Sí

No

Estadio del tumor: 


\section{Tabla 2. Variables e Instrumentos de medida}

\section{Variables de interés}

\section{Operativamente}

\section{Instrumento de evaluación}

Calidad de vida:

- Status de salud global

- Áreas funcionales:

Física

Social

Rol

Emocional

Cognitiva

- Escalas sintomáticas:

Astenia

Náuseas

Dolor

- Síntomas específicos:

Puntuación dada a

diferentes ítems que evalúan síntomas, áreas funcionales de calidad de vida y puntuación global
Escala QLQ-C30 de

EORTC (1993).

Versión española ${ }^{(5)}$

Disnea

Insomnio

Apetito

Estreñimiento

Diarrea

Economía

\begin{tabular}{lll}
\hline $\begin{array}{l}\text { Ansiedad } \\
\text { Depresión }\end{array}$ & $\begin{array}{l}\text { Puntuación dada a ítems } \\
\text { de ansiedad y depresión. }\end{array}$ & $\begin{array}{l}\operatorname{HAD}^{(10,)} \\
\text { Versión española }^{(11)}\end{array}$ \\
\hline Astenia & $\begin{array}{l}\text { Puntuación en ítems de } \\
\text { astenia general y de su } \\
\text { interferencia en la vida. }\end{array}$ & $\begin{array}{l}\text { Breve Inventario de } \\
\text { Fatiga }^{(14)}\end{array}$ \\
\hline
\end{tabular}

Déficit de hemoglobina en Hombre: menor a $13 \mathrm{~g} / \mathrm{dl}$. Hemograma sangre Mujer: menor a $12 \mathrm{~g} / \mathrm{dl}$.

$\mathrm{Dt}=9,19$; rango 38-85; el 58\% tienen entre 50 y 69 años y el 38\% más de 70 años), casado $(79,2 \%)$, con hijos $(85,7 \%)$; vive en pareja $(75,3 \%)$; dominancia manual diestra $(93,5 \%)$.

- Escolaridad y trabajo: el 39\% de los participantes tiene menos de cinco años de estudios, y el $61 \%$ restante tiene entre cinco y doce años de estudios o más, aunque el 54,5\% no llegó a alcanzar la titulación de EGB; trabajó por cuenta ajena $(44,2 \%)$ y actualmente está jubilado (55,8\%).

- Antecedentes clínicos: no presenta antecedentes de enfermedad neuropsicológica (93\%).

- Trastornos actuales: no refiere trastornos de salud mental $(87 \%)$ y comunica no tener problemas físicos relacionados con la enfermedad o con los tratamientos ni problemas 
psicosociales de gravedad. No necesita cuidador $(92,2 \%)$.

- Situación clínica actual: diagnosticado de cáncer de colon, estadio III $(65,7 \%)$, el tiempo desde la cirugía hasta que es evaluado es de un mes y medio. Su pronóstico es favorable (72,1\%).

Respecto a los resultados en las variables de interés:

- Estado de ánimo: según las puntuaciones en la Escala de Ansiedad y Depresión Hospitalaria (HAD), los pacientes de cáncer de colon muestran:

- Promedio de depresión de 3,7 sobre 21 puntos ( $\mathrm{Dt}=3,6$ y rango $0-18$ ). Esta puntuación se ubica en el rango de depresión no clínica. Con posterioridad a la cirugía, el 83,1\% (64 participantes) de los pacientes presentó puntuaciones en la escala depresión dentro del rango considerado normal (0-7), el 9,1\% (7 personas) fueron calificados como caso dudoso, y el $7,8 \%$ de la muestra (6 personas) obtuvo una puntuación clínica en dicha escala.

- Promedio de la ansiedad, de 4,5 sobre 21 puntos en ansiedad (Dt $=$ 3,47 y rango $0-17$ ). Respecto al porcentaje de casos clínicos, el 83,1\% de los pacientes (64 participantes) obtuvieron puntuaciones dentro del rango de la normalidad, el $10,4 \%$ se calificó como caso dudoso (8 personas) y el 6,5\% (5 personas) alcanzó un resultado clínico en ansiedad.

La astenia evaluada mediante el Breve Inventario de Fatiga (BIF), muestra los siguientes resultados:

- La muestra obtiene una astenia media general de 1,35 sobre $10(\mathrm{Dt}=$
1,96 y rango 0-8), mientras que la media de la interferencia de ésta en diferentes aspectos de la vida diaria es de 0,85 ( Dt $=1,54$ y rango 0-8). Tras el tratamiento quirúrgico, el promedio de astenia $(1,35)$ en los pacientes de cáncer de colon examinados se ubica en el rango de astenia bajo, un 92\% de la muestra tiene una puntuación baja, un $4 \%$ media y el $4 \%$ restante alta. El promedio de la interferencia de ésta con la vida diaria es igualmente baja $(0,85)$, un $96 \%$ de la muestra comunica tener una escasa interferencia de la astenia en su vida, $1,3 \%$ comunica interferencia media y un $2,6 \%$ alta interferencia.

Las puntuaciones en hemoglobina basal en sangre, evaluadas mediante un hemograma, son indicativas de anemia:

- La media grupal es de 12,41 g/dl. (Dt =1,48 y rango 9,1-15,6), ubicándose por debajo de 13 g/dl., punto de corte bajo el que se diagnostica anemia en hombres. En las mujeres $(n=24)$, la hemoglobina media es de 11,96 g/dl., ligeramente por debajo de 12 g/dl., punto de corte bajo el cual se diagnostica anemia. En este grupo, un 45,8\% (11 participantes) mostraron una cantidad de hemoglobina en sangre inferior a $12 \mathrm{~g} / \mathrm{dl}$. Mientras, la media en el grupo compuesto por hombres $(\mathrm{n}=$ 45) es de 12,65 g/dl., por debajo del punto de corte correspondiente, un $53 \%$ (24 participantes) presentaron niveles de hemoglobina en sangre inferiores a $13 \mathrm{~g} / \mathrm{dl}$.

Calidad de vida: en las tablas 3 y 4 podemos observar una comparación realizada entre las puntuaciones de los participantes en la escala QLQ-C30, después 
de resecar el cáncer, y los valores referenciales ofrecidos por dicha prueba para la población general europea de enfermos de cáncer de colon local (Scott et al., $\left.{ }^{(9)}\right)$. La tabla 3 compara la puntuación obtenida por la muestra en calidad de vida global y en las áreas funcionales con los valores de referencia, mientras que la tabla 4 realiza dicha comparación en cuanto a síntomas, con la prueba $t$ de Student.

Los resultados de la tabla 3 muestran como los pacientes evaluados presentan algunas puntuaciones por encima de los valores referenciales de forma estadísticamente significativa, siendo superiores los resultados a favor de la muestra en las áreas funcionales física, rol y social. Los datos revelan que las puntuaciones en calidad de vida son satisfactorias, ya que se aproximan a 100 en las escalas funcionales. La escala global es moderada y no se diferencia del dato ofrecido por el baremo.
En la tabla 4, hay que tener presente que una mayor puntuación refleja síntomas más intensos y, por tanto, mayor interferencia con la calidad de vida. Las puntuaciones más altas se dan en problemas de sueño y fatiga, ambos superan los 20 puntos sobre 100, el resto está por debajo de 15 puntos. Los pacientes que no han recibido quimioterapia puntúan igual a lo esperado en siete de nueve síntomas, según los valores referenciales europeos propuestos por el QLQ-C30 para pacientes con cáncer de colon. Las excepciones son el dolor y la disnea, los pacientes de la muestra parecen sentir menos dolor y disnea de lo planteado por el valor normativo correspondiente.

\section{DISCUSIÓN}

Tras la realización de la cirugía para la extirpación de cáncer de colon, son muy escasos los pacientes que tienen ansiedad

Tabla 3. Calidad de Vida: Global y Áreas Funcionales.

Puntuación pretratamiento vs. Valor referencial de muestras europeas con cáncer de colon local(9)

\begin{tabular}{|c|c|c|c|c|}
\hline \multicolumn{2}{|c|}{$\begin{array}{l}\text { Calidad de vida: } \\
\text { QLQ-C30 (N) }\end{array}$} & $\begin{array}{c}\text { Medida Pre: } \\
\text { Media (Dt) }\end{array}$ & $\begin{array}{l}\text { Valor } \\
\text { referencial }\end{array}$ & $\stackrel{t}{t}$ Sig. bilateral \\
\hline \multicolumn{2}{|c|}{ GLOBAL (77) } & 69,26 & 68,5 & 0,27 \\
\hline \multirow[t]{5}{*}{ Función } & Física (77) & $\begin{array}{c}88,13 \\
(14,89)\end{array}$ & 77,5 & $\begin{array}{c}6,26 \\
0,000^{* *}\end{array}$ \\
\hline & Rol (77) & $\begin{array}{c}82,68 \\
(29,17)\end{array}$ & 75,0 & $\begin{array}{c}2,31 \\
0,02 *\end{array}$ \\
\hline & Emocional (76) & $\begin{array}{c}77,96 \\
(19,70)\end{array}$ & 78,6 & $\begin{array}{l}-0,28 \\
0,77\end{array}$ \\
\hline & Cognitiva (77) & $\begin{array}{c}89,61 \\
(16,65)\end{array}$ & 87,4 & $\begin{array}{l}1,16 \\
0,24\end{array}$ \\
\hline & Social (77) & $\begin{array}{c}85,93 \\
(20,95)\end{array}$ & 68,5 & $\begin{array}{c}7,30 \\
0,000^{* *}\end{array}$ \\
\hline
\end{tabular}


Tabla 4. Calidad de vida: Síntomas.

Puntuación pretratamiento vs. Valor referencial de los

pacientes de cáncer de colon local en muestras europeas ${ }^{(9)}$

\begin{tabular}{|c|c|c|c|}
\hline $\begin{array}{l}\text { Síntomas } \\
(\mathrm{N})\end{array}$ & $\begin{array}{l}\text { Medida Pre: } \\
\text { Media (Dt) }\end{array}$ & $\begin{array}{c}\text { Valor referencial } \\
\text { QLQ-C30 }\end{array}$ & $\begin{array}{c}t \\
\text { Sig. bilateral }\end{array}$ \\
\hline Fatiga (75) & $\begin{array}{c}24,88 \\
(22,58)\end{array}$ & 29,6 & $\begin{array}{c}-1,80 \\
0,07\end{array}$ \\
\hline Náuseas (76) & $\begin{array}{c}3,07 \\
(12,38)\end{array}$ & 5,0 & $\begin{array}{c}-1,35 \\
0,17\end{array}$ \\
\hline Dolor (77) & $\begin{array}{c}11,90 \\
(17,69)\end{array}$ & 18,9 & $\begin{array}{c}-3,46 \\
0,001^{* *}\end{array}$ \\
\hline Disnea (77) & $\begin{array}{c}4,76 \\
(14,01)\end{array}$ & 9,0 & $\begin{array}{l}-2,65 \\
0,01 *\end{array}$ \\
\hline Insomnio (76) & $\begin{array}{c}25,87 \\
(30,11)\end{array}$ & 25,9 & $\begin{array}{l}-0,007 \\
-0,99\end{array}$ \\
\hline Apetito (77) & $\begin{array}{c}14,71 \\
(29,36)\end{array}$ & 13,2 & $\begin{array}{l}0,45 \\
0,65\end{array}$ \\
\hline Estreñimiento (77) & $\begin{array}{c}14,28 \\
(27,26)\end{array}$ & 11 & $\begin{array}{l}1,05 \\
0,29\end{array}$ \\
\hline Diarrea (77) & $\begin{array}{c}12,55 \\
(24,80)\end{array}$ & 11,7 & $\begin{array}{l}0,30 \\
0,76\end{array}$ \\
\hline Económico (77) & $\begin{array}{c}8,22 \\
(27,65)\end{array}$ & 12,1 & $\begin{array}{l}1,23 \\
0,22\end{array}$ \\
\hline
\end{tabular}

o depresión clínica (según el cuestionario HAD, seis personas presentaron niveles clínicos de depresión y 5 de ansiedad), el resto se encuadran dentro de la normalidad (64 participantes para ambas variables) o son casos considerados dudosos. Estos datos son mucho más positivos que los informados en los estudios que han aplicado el HAD, como puede verse en Soriano et al. ${ }^{(16)}$, en pacientes de cáncer de colon ostomizados, que ofrece datos de $19 \%$ de casos de depresión y $29 \%$ de casos de ansiedad, o en la revisión de Mitchell et al. ${ }^{(17)}$, con diversos tipos de cáncer que ofrecen cifras entre $30-40 \%$ de casos de prevalencia de ansiedad o depresión.
Se ha de considerar que en el presente trabajo se trata de pacientes sin cáncer avanzado, que no han recibido tratamientos de quimioterapia todavía, por lo que no han experimentado los efectos colaterales de estos tratamientos, al mismo tiempo que pueden mantener una expectativa positiva en las posibilidades de control de estos tratamientos en su enfermedad.

El grupo refiere astenia baja, igual que la influencia que ésta ejerce en distintas facetas de la vida cotidiana.

Como promedio, la muestra de pacientes tiene anemia ya que la cantidad basal de hemoglobina en sangre fue de $11.96 \mathrm{~g} / \mathrm{dl}$ en mujeres (normal $12 \mathrm{~g} / \mathrm{dl}$ ) y 
$12.6 \mathrm{~g} / \mathrm{dl}$ en el hombre (normal $13 \mathrm{~g} / \mathrm{dl}$ ). El $46 \%$ de las mujeres y el $53 \%$ de los hombres presentan anemia.

Antes de recibir el tratamiento prescrito, la calidad de vida global de los evaluados es moderada y similar a la de sus homólogos europeos. De las cinco áreas funcionales evaluadas por el QLQ-C30, las funciones física, rol y social superan lo esperable, y además los síntomas dolor y disnea son más bajos a lo estipulado.

Los datos de los estudios de seguimiento de la calidad de vida y síntomas físicos de pacientes de cáncer a un año -Arnd et al. ${ }^{(18)}$-, más cinco años -Jansen et al. ${ }^{(19)}{ }_{-}$, o a 10 años -Thong et al. ${ }^{(20)}$ demuestran que la calidad de la vida de los pacientes de cáncer es relativamente satisfactoria para la mayoría de ellos, tal como muestran los datos del presente estudio que incluso da resultados de calidad de vida, sintomatología y malestar emocional mejores que los informados en dichos trabajos.

Por consiguiente, pese al diagnóstico de cáncer de colon, sólo un subgrupo de pacientes que ya han sido operados para la extirpación de la masa cancerosa, presentan estados emocionales alterados; en concreto, menos del 10\% muestran ansiedad y depresión clínica. Así mismo, tan solo mes y medio después del tratamiento quirúrgico la astenia producida por la enfermedad y la cirugía se reduce, siendo bajos tanto el promedio de astenia como la interferencia de ésta con la vida del paciente, un $92 \%$ presenta astenia general baja y un 96\% escasa interferencia de ésta en actividades cotidianas. Las personas evaluados mantienen una calidad de vida buena, similar a la de los enfermos europeos de cáncer de colon local, pero respecto a estos, muestran una mejor calidad de vida en las áreas funcionales física, rol y social e incluso menor sintomatología, en concreto menos dolor y disnea. Únicamente se aprecia la existencia de anemia en la muestra, esto es así también cuando se valora el grupo de hombres y mujeres por separado, pudiendo padecer anemia en torno al $50 \%$ de la muestra. Si bien es predecible que dicha variable sea controlada periódicamente por los profesionales de la oncología con vistas a la aplicación del tratamiento sistémico, por lo que se presupone adecuada supervisión médica y evolución favorable.

\section{CONCLUSIÓN}

Este estudio arroja resultados optimistas, sólo un subgrupo de pacientes con cáncer de colon tiene problemas que atañen a su estado emocional y físico, con un porcentaje inferior al 10\% de estados clínicos de ansiedad y depresión o graves en el caso de la astenia. Por otro lado, tras un tiempo del tratamiento quirúrgico, alrededor de la mitad de los evaluados muestran un nivel de hemoglobina indicativo de anemia, variable que podría estar incidiendo negativamente en algún aspecto de la vida del paciente y por tanto se hace recomendable su tratamiento, reconociendo la importante labor de los oncólogos en su adecuada supervisión y tratamiento. La calidad de vida global de los pacientes de cáncer de colon españoles es similar a la de los homólogos europeos; algunas áreas funcionales y síntomas que puede alterar la calidad de vida son mejores a los valores de referencia. En definitiva, tras la cirugía el paciente oncológico presenta buen estado de ánimo, buena calidad de vida y escasa astenia, aunque puede presentar niveles bajos de hemoglobina en sangre.

\section{REFERENCIAS BIBLIOGRÁFICAS}

1. National Institute of Cancer (NIC). Colon and rectal cancer. [documento en Internet] Acceso el 6 de Agosto, 2007. Disponible en: http://www.cancer.gov/espanol 
2. Instituto de Salud Carlos III. (2005). Informe sobre la salud de los españoles.

Cáncer [Versión electrónica]. 2005. Acceso el 28 de octubre, 2007. Disponible en:

http://www.isciii.es/htdocs/centros/ epidemiologia/epi-cancer.jsp

3. Váquez, JMM, Docobo FD, Charlo TD. Estado actual del tratamiento quirúrgico del cáncer colorrectal. Cir Esp 2000; 68: 150-6.

4. Agra YV, Badía XL, Gil A.M. Instrumentos para la medición de la calidad de vida en los pacientes con cáncer. Med Clín 1998; 110: 703-8.

5. Arrarás.I, Illarramendi JJ, Valerdi JJ. El cuestionario de Calidad de Vida de la EORTC, QLQ-C30. Estudio estadístico de validación con una muestra española. Rev Psicol Salud 1995; 7: 13-30.

6. Arrarás JI, Dueñas T, Meiriño R, Prujá E, Villafranca E, Valerdi JJ. La Calidad de Vida en el paciente oncológico: estudios del Servicio de Oncología del Hospital de Navarra en el Grupo de Calidad de Vida de la EORTC. An Sist Sanit Nava 1998; 21(1):47-53.

7. Arrarás JI, Martínez, M, Manterota A. Laínez N. El grupo de la calidad de vida de la EORTC. Psicooncología 2004; 1: 8798.

8. Le AT, Albo D, Berger DH. Quality of life in the elderly with rectal cancer.J Am Coll Surg., 2007; 205: 124-31. Doi: 10.1016/j. jamcollsurg.2007.02.028.

9. Scott NW, Fayers PM, Aaronson N.K, Bottomley A, de Graeff A, Groenvold M et al. EORTC QLQ-C30 Reference Values. [Documento en internet] Acceso el 1 enero de 2009. Disponible en:

http://groups.eortc.be/qol/downloads/ reference_values_manual2008.pdf

10. Zigmond AS, Snaith RP. The Hospital Anxiety and Depression Scale. Acta Psychiatr Scand 1983; 67:361-70. Doi: 10.1111/j.1600-0447.1983.tb09716.x.

11. Comeche MIM, Díaz MIG., Vallejo MAP. Cuestionarios, inventarios y escalas: ansiedad, depresión y habilidades sociales. Madrid: Fundación Universidad-Empresa, 1995. p. 82-6.

12. Caro I, Ibáñez E. Escala hospitalaria de ansiedad y depresión. Su utilidad práctica en Psicología de la salud. Bol Psicol 2002; 36: 43-69.

13. Bower JE, Ganz PA, Desmond KA, Bernaards C, Rowland JH, Meyerowitz BE. et al. Fatigue in long-term breast carcinoma survivors. A longitudinal investigation. Cancer 2006; 106: 751-8. Doi: 10.1002/ cncr.21671.

14. Mendoza TR, Wang XS, Cleeland CS, Morrisey M, Johnson BA, Wendt JK, et al. The rapid assessment of fatigue severity in cancer patients: Use of the Brief Fatigue Inventory. Cancer 1999; 85: 1186-96. Doi: 10.1002/(SICI)1097$0142(19990301) 85: 5<1186:$ :AID CNCR24>3.0.CO;2-N.

15. Ordóñez, A., Feijoo, M. y González Barón M. Evaluación de la astenia. En: GonzálezBarón M, Lacasta MA, Ordóñez A, editores. Valoración clínica en el paciente con cáncer. Madrid: Médica Panamericana, 2006. p. 79-87.

16. Soriano M, Cantero R., Olivares ME, Cruzado JA, Delgado I, Balibrea JM et al. Efectos de la cirugía laparoscópica en pacientes de cáncer colorrectal: calidad de vida, estado emocional y satisfacción. Psicooncología 2007; 4(1): 121-32.

17. Mitchell AJ, Chan M, Bhatti $H$, Halton $M$, Grassi L, Johansen $\mathrm{C}$, et al. Prevalence of depression, anxiety, and adjustment disorder in oncological, haematological, and palliative-care settings: A meta-analysis of 94 interview-based studies Lancet Oncol 2011; 12(2): 160-74.

18. Arndt V, Merx H, Stegmaier C, Ziegler H, Brenner H. Quality of life in patients with colorectal cancer 1 year after diagnosis compared with the general population: A population-based study. J Clin Oncol 2004; 22 (23): 4829-36. Doi: 10.1200/ JCO.2004.02.018. 
19. Jansen L, Koch L, Brenner H, Arndt V. 20. Thong MSY, Mols F, Lemmens VEPP, Jan Quality of life among long-term ( $\geq 5$ years) Creemers G-J, Slooter GD, van de Pollcolorectal cancer survivors - Systematic Franse LV. Impact of chemotherapy on review Eur J Cancer 2010; 46 (16): 287988. Doi: 10.1016/j.ejca.2010.06.010. health status and symptom burden of colon cancer survivors: A population-based study. Eur J Cancer 2011; Doi:10.1016/j. ejca.2011.02.006. 
